\title{
MÁS ALLÁ DE LAS MORALES: CONTROVERSIA, LUCHA, GRIETAS. EL SUBCAMPO DE LOS ESTUDIOS LITERARIOS Y LA UNIVERSIDAD ARGENTINA (1958-2015)
}

\author{
BEYOND MORALS: CONTROVERSY, STRUGGLE, RIFTS. \\ THE SUBFIELD OF LITERARY STUDIES AND THE ARGENTINIAN \\ UNIVERSITY (1958-2015)
}

\section{ANALÍA GERBAUDO*}

Resumen: Este artículo se desprende del proyecto International Cooperation in the Social-Sciences and Humanities: Comparative Socio-Historical Perspectives and Future Possibilities, dirigido por Gisèle Sapiro. Dicha investigación buscó delinear una "morfología comparativa" que diera cuenta de la organización de las ciencias sociales y humanas en siete disciplinas (Sociología, Psicología, Filosofía, Economía, Literatura, Antropología y Ciencias Políticas) en nueve países (Argentina, Brasil, Francia, Italia, Reino Unido, Austria, Países Bajos, Hungría y Estados Unidos). En ese marco analicé los procesos de institucionalización e internacionalización de los estudios literarios en Argentina entre 1958 y 2015. Esta presentación se basa en la síntesis de esa investigación: examina el lugar que la universidad ha ocupado en un subcampo atravesado por la fluctuación entre dictaduras y restituciones de la democracia, crisis económicas y diferentes políticas de financiamiento de la educación, la ciencia y la tecnología. La primera sección precisa, sintéticamente, las decisiones metodológicas tomadas. Luego se resumen los resultados. Finalmente, se concluye con reflexiones sobre el lugar de la universidad en la dinámica del subcampo de los estudios literarios en Argentina.

Palabras clave: Argentina, campo, estudios literarios, universidad, formaciones.

Aвstract: This article originates from the project International Cooperation in the Social-Sciences and Humanities: Comparative Socio-Historical Perspectives and Future Possibilities directed by Gisèle Sapiro. Such project aimed at delineating a "comparative morphology" which accounted for the institutional organization and internationalization of Humanities and Social Sciences in seven disciplines (Sociology, Psychology, Philosophy, Economics, Literature, Anthropology, and Political Science) and in nine countries (Argentina, Brazil, France, Italy, the United Kingdom, Austria, the Netherlands, Hungary and the United States). Within that project I analyzed the processes of

\footnotetext{
* Doctora en Letras Modernas. Universidad Nacional del Litoral, Consejo Nacional de Investigaciones Científicas y Técnicas, Santa Fe, Argentina. Correo electrónico: analia.gerbaudo@conicet.gov.ar. Orcid: https://orcid.org/0000-0001-9969-8004.
} 
institutionalization and internationalization of literary studies in Argentina between 1958 and 2015. What follows is based on a synthesis of that study. This article examines the place the university has had in a subfield affected by fluctuation between dictatorships and restorations of democracy, economic crises and different public policies regarding the funding of education, science and technology. The first section briefly pinpoints the methodological decisions made in this study, followed by a brief reconstruction of the results. This paper concludes with reflections on the university and the dynamics of the subfield of Literary Studies in Argentina.

KEYwords: Argentina, field, Literary Studies, university, formations.

Recibido: 2019-04-19. Aceptado: 2020-01-29.

\section{INTRODUCCIÓN}

- Ste artículo parte de los resultados finales de una investigación deUsarrollada en el marco del proyecto International Cooperation in the Social-Sciences and Humanities: Comparative Socio-Historical Perspectives and Future Possibilities (INTERCO SSH) dirigido por Gisèle Sapiro. El estudio abarcó diferentes países (Argentina, Brasil, Francia, Italia, Reino Unido, Austria, Holanda, Hungría y Estados Unidos) y disciplinas (Sociología, Psicología, Filosofía, Economía, Letras, Antropología y Ciencias Políticas). Sapiro pretendió delinear una "morfología comparativa" que diera cuenta de la institucionalización y de la internacionalización de este recorte de las ciencias sociales y humanas entre 1945 y 2010. Algunos de los datos recogidos se utilizan aquí para abordar un problema puntual: se describe el rol de la universidad argentina en la dinámica del subcampo ${ }^{1}$ de los estudios literarios entre 1958 y $2015^{2}$.

El período se recorta entre dos "ciclos" (Beigel, 2016) de apoyo estatal a la ciencia y a la educación. El primer ciclo va desde 1958 hasta 1966, es decir, desde el año de fundación del Consejo Nacional de Investigaciones Científicas y Técnicas (CONICET), el más prestigioso organismo de investigación del país, y de EUDEBA (una de las editoriales universitarias que marcarán una tendencia en el campo editorial e intelectual) hasta el año del golpe de Estado liderado por Juan Carlos Onganía. El segundo ciclo va

\footnotetext{
${ }^{1}$ Los subcampos de los estudios literarios, lingüísticos y semióticos integran el campo de las letras (para la distinción entre campos y subcampos, ver Bourdieu, 1997, 2001a).

${ }^{2}$ Una vez concluido el proyecto INTERCO SSH (2012-2017) modificamos el período propuesto por Sapiro: analizamos cómo se configura el campo de las letras entre dos momentos excepcionales para su institucionalización debido a las políticas públicas.
} 
desde 2004 a 2015: durante estos años se desarrollan políticas de Estado que fortalecen y expanden tanto el sistema educativo como el científico a través de la creación de nuevas universidades públicas, la inversión en infraestructura, el aumento de la cantidad de carreras de doctorado y de becas del CONICET destinadas a realizar estudios doctorales en el país, la repatriación e incorporación de nuevos científicos al CONICET, la promoción de la internacionalización, etc. Durante las últimas elecciones de 2015 triunfa una coalición de derecha que, apenas asume el gobierno, interrumpe estas políticas públicas (Sorá y Beigel, 2019, p. 328). Lo acontecido en y entre estos dos ciclos en el subcampo de los estudios literarios se examina a la luz de los procesos de su institucionalización, de su autonomización y del "desarrollo profesional" de sus agentes.

En primer lugar, se exponen algunas de las decisiones metodológicas tomadas para reconstruir cómo se institucionalizan las letras en Argentina y cómo se internacionalizan: los datos recogidos para el análisis del proceso de internacionalización, relevados durante la segunda etapa del proyecto, permiten completar aspectos del proceso de institucionalización relevados durante la primera etapa. En segundo lugar, se caracteriza el proceso de institucionalización de los estudios literarios mientras se describe el de autonomización relativa del subcampo y de desarrollo profesional de sus agentes: en ese marco complejo la universidad, el CONICET y las "formaciones" (Williams, 1977) jugarán roles convergentes o divergentes según las coyunturas. Finalmente, se esbozan algunas conclusiones respecto de las marcas dominantes del subcampo de los estudios literarios en el período y su dinámica.

\section{CONSIDERACIONES METODOLÓGICAS}

Para reconstruir el proceso de institucionalización de las letras en Argentina seguimos las dimensiones de análisis propuestas por Sapiro al diseñar el proyecto INTERCO SSH. No obstante, tanto al momento de recoger los datos como de analizarlos fue necesario realizar ajustes en función de las particularidades de un campo situado en un país periférico atravesado por la violencia política estatal ${ }^{3}$.

\footnotetext{
${ }^{3}$ Este concepto se desprende de los cursos dictados por Pierre Bourdieu entre 1989 y 1992 en el Collège de France (cf. Bourdieu, 2012).
} 
Las dimensiones de análisis propuestas por Sapiro (2012) para reconstruir los "índices de institucionalización" son cuatro: 1. enseñanza (fecha de creación de la carrera, número de ingresantes por año, número de profesores por disciplina [\% de mujeres, $\%$ de extranjeros, $\%$ de doctores]); 2 . Investigación (instituciones de investigación y acuerdos, instituciones no académicas y lugares de investigación); 3. publicaciones (creación de revistas científicas en la disciplina (fecha, perfil), creación de revistas temáticas e interdisciplinarias, colecciones editoriales especializadas); 4. organización profesional (creación de sociedades académicas o asociaciones profesionales en la disciplina [fecha, número de miembros, categorías], mecanismos de evaluación pública y de distinción, mercado de trabajo).

El análisis de casos nos permitió escribir en un tiempo breve un primer informe sobre la institucionalización de las "letras" (cf. Gerbaudo, 2014) en un país con archivos ${ }^{4}$ precarios que, en el arco temporal recortado, modifica notablemente su cartografía universitaria (cf. CONEAU, 2012). Seleccionamos cuatro universidades: tres situadas en el "polo dominante" del campo y una en el "polo dominado" (Bourdieu, 2001a). Elegimos 4 universidades entre las más antiguas de Argentina: precisar los índices de institucionalización en la Universidad de Buenos Aires (UBA, 1821), la Universidad Nacional de La Plata (UNLP, 1890), la Universidad Nacional de Rosario (UNR, 1968) ${ }^{5}$ y la Universidad Nacional del Litoral (UNL, 1919) permite realizar análisis comparativos en un mismo corte temporal.

\footnotetext{
${ }^{4}$ Según Jacques Derrida (1995), la "domiciliación” es una de las condiciones para rotular a un texto como "archivo": "No hay archivo sin un lugar de consignación" (p. 26). Otra condición es su preservación en un soporte resistente. En este sentido, solo algunos de los documentos consultados para esta investigación cumplen con al menos una condición; excepcionalmente algunos, con las dos. A esto se suma la escasa fiabilidad de ciertas fuentes. Por ejemplo, el número de ingresantes a las carreras de Letras por universidad en ciertos períodos varía según la información la provea la propia universidad (cuando cuenta con los datos) o el Ministerio de Educación. Otro obstáculo es el que origina la destrucción deliberada o accidental de documentos y la inexistencia de datos sobre ciertos tramos: esto obligó a una reconstrucción artesanal de cada una de las informaciones compiladas en las tablas de nuestro primer informe. Por ejemplo, para reconstruir el número de ingresantes a la carrera de Letras de la Universidad Nacional del Litoral, se tuvo que revisar, uno por uno, cada dossier de cada estudiante en la sección Alumnado (Gerbaudo, 2014, p. 34). Por estas razones presentamos los datos que ponemos a disposición allí con carácter de "borrador" (Gerbaudo, 2014, p. 18). Nos anima un comentario que Bourdieu desliza durante una entrevista con Yvette Delsaut: contra el "efecto de cierre" que producen ciertos textos y contra la desaparición de las "huellas del trabajo en beneficio de lo definitivo, de lo terminado", Bourdieu destaca los beneficios de la "lógica del borrador" en la producción de conocimiento científico mientras defiende "el derecho de publicar textos no definitivos" (2001b, p. 198).

${ }^{5}$ La UNR se funda en 1968. Es un desprendimiento de la UNL. El polo Rosario, ineludible en cualquier análisis de la institucionalización de las letras en Argentina, pasa entonces de la UNL a la UNR.
} 
Ahora bien, al momento de volcar la información sobre las cuatro dimensiones citadas en planillas y tablas ordenadas por institución, advertimos la necesidad de dar cuenta de lo acontecido en los espacios que Raymond Williams llama "formaciones" (1977): relevamos las acciones realizadas fuera de toda institución estatal durante y entre las dos últimas dictaduras por agentes que habían trabajado y/o estudiado en estas cuatro universidades. Este tipo de información, relevada por Sapiro en su análisis de la configuración del campo literario francés durante la ocupación alemana (1999), necesita incluirse en el análisis de la configuración del campo de las letras en Argentina por dos razones: 1) son las formaciones las que garantizan su autonomía relativa frente a las coacciones estatales; 2) algunas áreas no fueron afectadas en su desarrollo institucional en los períodos de violencia política mientras que otras solo pudieron desarrollarse en formaciones. Por ejemplo, durante las dictaduras, la filología y los estudios clásicos continuaron sus desarrollos "académicos" (Funes, 2009, 2011, p. 97) ${ }^{6}$.

Finalmente, la dimensión de análisis "organización profesional" (Sapiro, 2012) que comprendía al "mercado de trabajo" exigía recolectar datos que precisaran sus cambios atendiendo tanto a las políticas públicas como a los obstáculos para investigar y para enseñar oficialmente ciertas líneas teóricas en el arco 1958-2015. Debido a la cantidad de información requerida para este análisis, lo postergamos para retomarlo en la segunda etapa: la construcción de una base de datos para el estudio del proceso de internacionalización nos permitió dar cuenta de esta dimensión.

Respecto del proceso de internacionalización, si bien este artículo no lo examinará, se recurrirá a datos relevados en su reconstrucción que aportan al problema aquí tratado. Por lo tanto, es necesario observar, en primer lugar, que las dimensiones de análisis seguidas se desprenden de investigaciones previas (Bourdieu, 2002; Heilbron et al., 2009; Heilbron y Gingras, 2009; Sapiro, 1999; Boschetti, 2009) y de los criterios propuestos por Sapiro para el proyecto INTERCO SSH (Sapiro et al., 2013). Fijamos cuatro

\footnotetext{
${ }^{6}$ Algunos datos sobre las intromisiones estatales en la universidad y en el CONICET durante la última dictadura: en 1976 el presidente de facto, Jorge Rafael Videla, declara que la "subversión" había encontrado en las universidades un "campo propicio para su desenvolvimiento" (Avaro, 2015, p. 94). Congruente con este punto de vista, el presupuesto universitario se achica (entre 1975 y 1976, pasa del $26 \%$ al 8\%). Como contrapartida, el presupuesto del CONICET se expande (entre 1975 y 1976 pasa del $13 \%$ al 26\%) (Bekerman, 2013, p. 251). Esta transferencia de recursos se explica a partir de dos prácticas complementarias: "el fuerte disciplinamiento" en el CONICET (un organismo fácilmente controlable dada su organización interna y su reducido número de agentes) se combina con la persecución, despido y desaparición de profesores y estudiantes universitarios (cf. CONADEP, 1984: pp. 31, 103 y 213).
} 
dimensiones: 1. Migraciones; 2. Cooperación; 3. Publicaciones; 4. Traducciones.

En segundo lugar, el análisis combina un doble plano: por un lado, el de los datos empíricos, orientado por las dimensiones citadas; por el otro, el de la "intencionalidad de los actores" pretación de los resultados cuantitativos sobre migraciones, cooperación internacional, publicaciones en el extranjero, intraducción y extraducción extraídos de una base de datos construida con currículums de 181 agentes del subcampo (112 mujeres; 69 varones) se combina con una interpretación de las respuestas a una entrevista semiestructurada a dichos agentes y con consultas vía mail. Se realizaron 148 entrevistas (96 mujeres; 52 varones) ${ }^{8}$. Datos y "cuentos" se combinan a los efectos de enriquecer el análisis de los procesos de institucionalización de los estudios literarios en Argentina y de su internacionalización". Si bien el concepto de "cuento" se desarrolla en otro lugar donde se lo rodea de observaciones teóricas para advertir respecto de las precauciones epistemológicas necesarias en el uso de información tomada de relatos ${ }^{10}$, vale aclarar que el término subraya el carácter parcial y subjetivo de la información recogida a través de entrevistas y consultas. Por lo tanto, en esta investigación los "cuentos" se utilizan para: 1) reconstruir procesos históricos sobre los que no hay archivo; 2) analizar la relación entre prácticas del agente y tomas de posición sobre dichas prácticas.

En tercer lugar, los datos se interrogan a partir de la adaptación de los criterios seguidos por Sapiro (1999) en su estudio del campo literario francés bajo la ocupación alemana. Su decisión de conjugar la carrera profesio-

\footnotetext{
${ }^{7}$ Retomo la expresión usada por Louis Pinto al aconsejarme incluir este aspecto en el análisis. Esta incorporación capitaliza los resultados de una investigación, ya entonces en curso y continuada hasta el presente, centrada en el análisis de las fantasías de nano-intervención de los críticos que enseñaron Teoría literaria y Literatura argentina en la universidad pública de la posdictadura (Gerbaudo, 2016). Reconstruir estas fantasías tanto a partir de los relatos de los agentes como a partir de sus acciones potencia el análisis de la relación entre lo deseado desde el plano "individual" y lo posible en el campo (Bourdieu, 2001a).

${ }^{8}$ La diferencia entre el número de agentes incluidos en la base de datos y el número de agentes que respondieron la entrevista obedece a dos razones: 1) algunos curriculums debieron reconstruirse en base a la información disponible debido a que los agentes habían fallecido al momento de realizar esta investigación; 2) por diversos motivos, no todos los agentes pudieron ser entrevistados.

${ }^{9}$ El esquema de la entrevista se incluye en el primer informe producido en el marco de esta investigación, disponible on line (Gerbaudo y Fumis, 2014, p. 259).

${ }^{10}$ Para el concepto de "cuento", su alcance probatorio restringido y su importancia para complejizar los resultados derivados del análisis cuantitativo, envío a un texto disponible on line en acceso abierto, donde también se precisa el concepto de "consulta", se lo diferencia del concepto de "entrevista" y se justifica por qué, en esta investigación, se incluyen los nombres de los agentes entrevistados y consultados (Gerbaudo, 2018a).
} 
nal ${ }^{11}$ de los agentes con factores "extraliterarios" (Sapiro, 1999, p. 706) es útil para analizar un subcampo relativamente autónomo, continuamente jaqueado por la discontinuidad de las políticas públicas, por el terrorismo de Estado durante buena parte de la segunda mitad del siglo XX y por las crisis económicas. Se atiende, entonces, a la edad que tenían los agentes durante las dos últimas dictaduras y durante la década del noventa, marcada por el modelo neoliberal que derivó en el estallido social de diciembre de 2001. Esta decisión metodológica apunta a verificar la correlación entre dictaduras, crisis económicas e internacionalización. De este modo, los datos tomados tanto de los curriculums como de las entrevistas se ordenan según cinco grupos de agentes:

Grupos de agentes según su edad (tomada en 2015):

-Grupo 1 (G1): desde 72 años

-Grupo 2 (G2): entre 62 y 71 años

-Grupo 3 (G3): entre 56 y 61 años

-Grupo 4 (G4): entre 40 y 55 años

-Grupo 5 (G5): entre 30 y 39 años.

Remarquemos que 1966 (año de inicio de la dictadura encabezada por Onganía y extendida hasta 1973) y 1976 (año de inicio de la última dictadura extendida hasta 1983) son fechas clave. No obstante, estas dictaduras se inscriben en un marco de "continuidad relativa en lo que respecta a la implantación de políticas represivas" (Franco, 2012, p. 18) con sus derivas

\footnotetext{
${ }^{11}$ En Profession? Écrivain, Sapiro (2017) examina las actuales condiciones para el desarrollo profesional de los escritores en Francia. El título del libro anticipa sus conclusiones: su investigación revela la tensión entre la profesionalización de la actividad de "escritor" y la "precarización creciente" de los autores (p. 7). Para ello se vale de datos tomados de entrevistas a los escritores que escudriña desde indicadores cualitativos a los efectos de "poder comparar las trayectorias" y caracterizar las "modalidades de ejercicio de la actividad" (p. 12). Seguimos su metodología y los conceptos de los que se deriva: "desarrollo profesional" y "trabajo precario" (Sapiro, 2017). El primero nos permite analizar las prácticas de los agentes con relación a las tendencias (autónomas/heterónomas) del campo de las letras: se trata de un campo relativamente autónomo respecto de otros (estatal, económico, militar, político, religioso, mediático) que lo impactan. El segundo concepto nos permite precisar, en diálogo con el primero, los indicadores a tomar en cuenta para hablar de precarización laboral, a saber: 1) analizar si el agente logra sostenerse económicamente a partir de su actividad profesional (1.a. si la respuesta es positiva, diferenciar si le permite concentrarse en su línea de investigación o si debe diversificarla o abandonarla; en cada caso, verificar las causas; 1.b. si la respuesta es negativa, verificar qué otros trabajos realiza; verificar las causas); 2) analizar continuidades y cambios de situación en cada trayectoria laboral; precisar causas en ambos casos; 3 ) verificar la correlación entre situación laboral y producción científico-cultural en cada trayectoria atendiendo a continuidades/discontinuidades.
} 
sobre los procesos de institucionalización e internacionalización. Para mayor claridad, se precisa la edad de los agentes de cada grupo (en adelante, "G") al momento de estos acontecimientos:

-G1: agentes cuyas carreras estuvieron atravesadas por las dos últimas dictaduras (tienen, por lo menos 23 años en 1966 y 33 años en 1976);

-G2: agentes con por lo menos 23 años en 1976 (la formación de grado universitaria en Argentina dura 5 años, el nivel secundario se concluye a los 17 años y la duración promedio de la formación superior es de 5 a 6 años);

-G3: agentes con 22 años como máximo en 1976 y con 17 años como mínimo (a los 17-18 años se concluye la formación secundaria);

-G4: agentes con 16 años como máximo en 1976 y 23 años en 1983 (el punto de partida a los 40 años responde a las edades pautadas hasta 2014 para el ingreso a la Carrera de Investigador del CONICET: 40 años era la edad límite para la entrada directa a la categoría de Adjunto ligada a la generación de una línea de trabajo propia y a la formación de recursos humanos en el campo [Girbal, 2007, p. 372]).

-G5: agentes cuyo desarrollo profesional no se ve afectado sino de modo indirecto por las prácticas residuales de las dictaduras (tienen como máximo 7 años en 1983) y, de modo directo por las políticas neoliberales (tienen 25 años como máximo cuando se produce la crisis económica de 2001; 35 años era la edad límite para el ingreso a la Carrera de Investigador del CONICET como Asistente, primera categoría de la base de la pirámide, según la reglamentación vigente hasta 2014 [Girbal, 2007, p. 372]; 30 años es la edad promedio de fin de los doctorados).

Despejados los criterios y las razones que fundamentan las decisiones teóricas y metodológicas tomadas, en el apartado siguiente se condensan los principales resultados obtenidos que aportan al análisis del problema planteado en este artículo.

\section{LA UNIVERSIDAD EN EL SUBCAMPO DE LOS ESTUDIOS LITERARIOS (ARGENTINA, 1958-2015)}

Para presentar los resultados respecto de cómo interactúan instituciones (universidad y CONICET) y formaciones en la autonomización relativa del 
subcampo de los estudios literarios en Argentina y en el desarrollo profesional de sus agentes, sigo las cuatro dimensiones correspondientes al escudriñamiento del proceso de su institucionalización.

Enseñanza:

a) Durante y entre las dictaduras, como consecuencia de las desinstitucionalizaciones causadas por la violencia estatal (cf. Gerbaudo, 2014), los agentes crean los "grupos de estudio" privados y clandestinos también conocidos como "universidad de las catacumbas" (cf. Caisso y Rosa, 1987). Situados en los polos dominantes del subcampo (Buenos Aires, Rosario), permiten darle continuidad, en ocasiones de forma subterránea, a líneas excluidas de las instituciones. Lo producido en ellas marca, a mediano y a largo plazo, tanto la agenda como las prácticas de enseñanza en esas zonas cuyo desarrollo se ve interrumpido por las dictaduras (cf. Gerbaudo, 2016).

b) Paradójicamente, los agentes expulsados de las instituciones durante los tiempos de violencia política estatal terminarán fortaleciéndolas, en el caso de que estas los incorporen en otros momentos. Estas instituciones estatales aprovecharán los capitales simbólico, científico, cultural y social acumulados por los agentes, ya sea en el exilio, ya sea en formaciones. Por ejemplo, Susana Romano Sued (G2), detenida en los campos de concentración clandestinos de Córdoba durante la última dictadura, logra exiliarse en Alemania donde subsiste trabajando, en un primer momento, como peluquera. Luego cursa y finaliza su carrera doctoral en ese país. Regresa a la Argentina con la reinstalación democrática. Concursa la cátedra de Estética en la Universidad Nacional de Córdoba donde institucionaliza teorías que importa: enseña a Itamar Even Zohar y funda la línea de investigación en traducción en Argentina.

c) Se observa una expansión institucional asimétrica entre los polos dominados y dominantes del campo. Un indicador de esa asimetría es la fecha de creación de las carreras de Doctorado: mientras que en la UNL recién en 2011 están dadas las condiciones para crear un Doctorado en Humanidades, la UBA reorganiza su Doctorado en 1987 (es decir, apenas tres años después de restaurada la democracia), la UNLP hace lo propio con el suyo (creado con otros parámetros en 1920) y la UNR crea su Doctorado en Humanidades y Artes en 1998. 
Investigación:

a) Ciertas líneas del subcampo de la investigación literaria no pudieron desarrollarse en marcos institucionales durante y entre las dictaduras y durante ciertos momentos del primer ciclo de la posdictadura (1984-2003 $)^{12}$ debido al control ideológico estatal. Otras líneas fueron abandonadas por los agentes debido a los efectos "normalizadores" (Foucault, 1971) provocados por el disciplinamiento: la censura también llevó a la autocensura. Durante el primer ciclo de la posdictadura, el control ideológico estatal tuvo grados diferenciales según las instituciones y según los gobiernos que ocuparon el Estado: por un lado, las universidades pudieron construir espacios más auto-regulados que el CONICET, en parte debido a su reglamentación, que hace de la "autonomía" uno de sus principios fundamentales. Por otro lado, los controles ideológicos en el CONICET se observan durante el segundo y tercer momento del primer ciclo posdictatorial, es decir, durante "los años de Menem" (Pucciarelli, 2011). Algunos ejemplos: Norma Desinano (G1), integrante de los equipos de investigación universitarios dirigidos por Adolfo Prieto (G1) en Rosario, abandonará la línea de la sociología de la literatura argentina a partir de la dictadura de Onganía. Sus trabajos viran de los estudios literarios (1965) hacia los estudios lingüísticos orientados a problemas de enseñanza y de aprendizaje (cf. Desinano, 1974, 1988, 1995, 1997). Carmen Perilli (G2), profesora de Literatura Latinoamericana en la Universidad Nacional de Tucumán, cuenta que durante la última dictadura completaba parcialmente la información requerida por el CONICET en los incisos correspondientes a datos personales para evitar intromisiones de la Secretaría de Inteligencia del Estado. Su relato anuda esta situación con la dificultad para producir conocimiento en su área: ¿sobre qué temas, autores o problemas no era conveniente escribir a los efectos de no poner en riesgo la propia vida?, ¿qué bibliografía no se podía citar? (entrevista personal, 2014, inédita). Gustavo Bombini (G4), actualmente profesor en la UBA y en la Universidad Nacional de San Martín, pierde su beca doctoral del CONICET por usar en sus investigaciones sobre la enseñanza de la literatura en la escuela secundaria "bibliografía parcial e ideológica" (Bombini, 2004, p. 9). Esta descalificación obtusa se enunciaba en 1991 (7 años después de la restitución democrática) y se aplicaba a la obra de Adriana Puiggrós, referencia insoslayable en todo estado de la cuestión sobre la historia de la

\footnotetext{
${ }^{12}$ Para los diferentes "momentos" del primer ciclo de la posdictadura, ver Gerbaudo, 2016, pp. 94 108; para el nuevo ciclo de la posdictadura iniciado en 2016, ver Gerbaudo 2018b.
} 
enseñanza en Argentina. El año siguiente Ana Porrúa (G4), hoy investigadora del organismo y profesora de la Universidad Nacional de Mar del Plata (UNMdP), padece una situación similar. Su relato contrasta la autonomía de su universidad frente a los mecanismos oscuros del CONICET de este momento del primer ciclo de la posdictadura:

En 1992, durante el gobierno de Menem, cuando Matera estaba a cargo de la Secretaría de Ciencia y Técnica, quedé afuera del CONICET, como muchos otros becarios, con un dictamen extemporáneo sobre un informe de Iniciación que ya estaba aprobado. Un dictamen extemporáneo y escandaloso. En ese dictamen, que se publicó parcialmente en algunos diarios (recuerdo una nota en Tiempo Argentino o en Sur, no lo tengo a mano) se decía, entre otras cosas, que yo no hacía crítica literaria sino ideología, porque trabajaba la poesía de Ernesto Cardenal y Roque Dalton. En el mismo año, obtuve una beca de Perfeccionamiento en la UNMdP, con la que llevé adelante parte del doctorado (En entrevista por Santiago Venturini, 2015, inédita).

Publicaciones:

a) Frente a la violencia política estatal, tanto ideológica como económica, se crearon formaciones que continuaron, por medios privados, desarrollos iniciados en espacios institucionales públicos. Por ejemplo, el trabajo iniciado en EUDEBA por Boris Spivacow es continuado en la editorial que funda luego del golpe de Estado de Onganía: el Centro Editor de América Latina (CEAL).

b) Las publicaciones culturales marcan la agenda de ciertas líneas del subcampo de los estudios literarios. Por ejemplo, la revista central para la crítica literaria argentina entre fines de los setenta y hasta bien entrado el siglo XXI fue Punto de vista, situada deliberadamente al margen de los protocolos de las publicaciones científicas. Alberto Giordano (G3) y José Luis De Diego (G3) reconocen que mientras existió, una de sus aspiraciones mayores era publicar en Punto de vista: "Hasta hace poco tiempo, cuando dejó de salir, mi máxima aspiración como crítico era publicar en Punto de vista (lo conseguí en cuatro ocasiones, y fracasé en otras tantas)", confiesa Giordano (2011, p. 14). Por su parte, De Diego describe la revista como "el" espacio de circulación deseado: "soñábamos con publicar en Punto de vista" (entrevista personal, 2015, inédita). En ninguna de las 148 entrevistas recogidas en la muestra construida para esta investigación una revista científica tiene una valoración semejante. 
c) Apenas diez años después de la emergencia en Buenos Aires de la editorial Eloísa cartonera y del "arte post-crisis" (Giunta, 2009), en tres universidades públicas se crean editoriales "cartoneras": La Sofía cartonera se funda en 2012 en la Universidad Nacional de Córdoba; luego se crea Rita cartonera en la Universidad Nacional de Rosario y en 2015 se lanza el proyecto Vera cartonera en la Universidad Nacional del Litoral. Las tres editoriales son dirigidas por mujeres. Las dos primeras publican solo literatura: siguen la impronta del escritor Washington Cucurto que funda Eloísa... en 2003; la última, Vera cartonera, incluye textos de divulgación científica y se instala en un espacio institucional compartido entre la UNL y el CONICET. Los proyectos editoriales cartoneros no solo garantizan el bajo costo de los libros, sino que los apartan de los circuitos comerciales y ortodoxos tanto de su producción como de su circulación. Buscan acercar lo que la universidad produce a sectores sociales que han naturalizado su exclusión del circuito de consumo de ciertos bienes simbólicos (por ejemplo, se busca llegar a sujetos que nunca han entrado a una librería). También se busca asegurar, en una sociedad empobrecida, la posibilidad de acceso a bienes culturales de calidad a la población en general, incluidos los estudiantes universitarios. Se recrea, de este modo, una consigna tomada de otra editorial universitaria, EUDEBA, fundada durante el primer ciclo de expansión de la ciencia y la educación argentinas: "Libros para todos". Se trata de una consigna que Spivacow sostuvo luego en el CEAL durante las dos últimas dictaduras, a pesar de las bombas, las amenazas, la violencia contra sus trabajadores, la quema de libros: "Más libros para más". Las editoriales cartoneras toman elementos de los campos artístico (literario), editorial, académico y, en el caso de Vera, del científico, para construir un proyecto de "pequeña escala" (Bourdieu, 1999) alrededor de un nuevo objeto: el libro cartonero.

d) Las publicaciones de los agentes muestran tensiones del subcampo: por un lado, se verifica una tensión entre los protocolos del campo científico (Bourdieu, 1997) y los protocolos del campo artístico (Bourdieu, 1992, 2013) con sus respectivos habitus. Por el otro, se verifica una tensión entre dos culturas evaluativas institucionales correspondientes a dos instituciones diferentes: mientras que la universidad pone el foco de sus evaluaciones en la docencia, el CONICET pone el foco en las publicaciones aplicando criterios internacionalizados en constante ajuste, dominados por los parámetros de las ciencias naturales. Los efectos de esta "heterogeneidad estructural" (Beigel, 2016) se verifican en las prácticas de los agentes: más concretamente, en la lengua, género, soportes, formatos y circuitos elegi- 
dos para publicar. Por ejemplo, algunos agentes escriben solo en español ("una de las lenguas del conocimiento", insiste Daniel Link G3); otros, más radicales, usan una variedad rioplatense atravesada por expresiones de las lenguas de los pueblos originarios y/o del registro criollo (Camblong G2). Algunos agentes optan por el ensayo (Schvartzman G2; Camblong G2; Giordano G3; Amante, Oubiña, Mattoni G4, Surghi G5) frente al paper. Algunos agentes traducen a libro impreso en papel sus textos de Facebook (Giordano G3), sus blogs (Link G3), sus diarios (Ludmer G1) o sus notas de viaje (Sarlo G1): la mayoría de estos agentes son o han sido investigadores del CONICET, ocupan posiciones dominantes en el subcampo y pertenecen a los G1, G2 y G3, es decir, se trata de agentes con un capital simbólico acumulado que les permite sostener institucionalmente esta forma de activismo.

Organización profesional:

a) Si bien el trabajo precario es una constante, inscripto al menos en una etapa de todas las trayectorias de los agentes incluidos en la muestra, tiene marcas diferenciales en tanto responde a dos formas de violencia política estatal: la que se produce por efecto del terrorismo de Estado y la que se produce por efecto de las crisis económicas, resultado de la aplicación de políticas neoliberales.

b) A la precariedad laboral, los agentes oponen la inversión de fondos propios o de su salario para continuar con su desarrollo profesional que, en estos casos, toma la forma de un activismo, de una práctica de resistencia.

\section{DETERMINISMOS NO DETERMINISTAS (A MODO DE SÍNTESIS)}

Para introducir algunas de las conclusiones derivadas de lo expuesto, cito un fragmento de la respuesta de Geraldine Rogers (G4) a la entrevista diagramada para esta investigación. Rogers inscribe la palabra "inestabilidad" como marca estructural del subcampo de los estudios literarios en Argentina y arriesga sus derivas estructurantes en las prácticas de sus agentes:

Creo que muchos de quienes nos formamos en la universidad argentina de las últimas décadas tenemos un itinerario más ecléctico de lo que cualquier escuela académica autorizaría, y más contradictorio de lo que se requeriría para exhibir coherencia epistemológica y metodológica 
sostenida. En tren de ser optimista, también es posible que ese mismo eclecticismo -derivado de la condición indigente de nuestras letras- no sea solo una desventaja. Saber de bricolage para una producción en condiciones tácticas, más que estratégicas. (Entrevista por Daniela Gauna, 2016, inédita)

Rogers apela al lenguaje técnico de la guerra para pasar de la descripción de las condiciones de producción en el subcampo de los estudios literarios en Argentina a los resultados generados a partir de ellas. Retomo su diagnóstico como una suerte de prolegómeno a las conclusiones derivadas del análisis de los procesos de institucionalización de los estudios literarios en Argentina, de autonomización relativa del subcampo y de desarrollo profesional de sus agentes. A saber:

a) Los ciclos de expansión y de retracción de la inversión estatal en ciencia y educación unidas a la persecución ideológica generan, entre otras consecuencias: 1) el estancamiento parcial de la investigación en ciertas líneas durante las dictaduras y ciertos momentos del primer ciclo de la posdictadura; 2) la diversificación de objetos de investigación debido a las precarias y/o cambiantes circunstancias laborales (ya sea por protección, por pluriempleo o por las ofertas de trabajo durante el exilio); 3) la discontinuidad de los ritmos de institucionalización; 4) el refuerzo de la expansión institucional asimétrica con la consiguiente ratificación de los polos dominantes y dominados del campo.

b) La precariedad laboral y material (ausencia de infraestructura, equipamiento, bibliotecas actualizadas, etc.), prácticamente una constante en Argentina, no tiene correlato en procesos de desprofesionalización. Esto obedece a que buena parte de los agentes asocia la intervención intelectual con la resistencia política y/o el activismo. Esto motiva la inversión de fondos propios en la adquisición de bibliografía y equipamiento, asistencia a congresos, subvención de trabajo de campo, formación, etcétera.

c) Dos causas principales inciden en las prácticas y en la toma de posición de los agentes respecto de la institucionalización de los estudios literarios: 1) la coexistencia de dos culturas evaluativas con criterios no convergentes promovidas por la universidad y por el CONICET. Esta tensión es lo que Beigel (2016) denomina "heterogeneidad estructural"; 2) la conjunción de protocolos y habitus provenientes de los campos artístico (literario), universitario, científico y político (si es que allí inscribimos a las formaciones). 
En definitiva, los resultados dan cuenta de los determinismos no deterministas. Las grietas ${ }^{13}$ entre diferentes instituciones y entre estas y las formaciones habilitan lugares de intervención política en el sentido "nano": "más allá de" 14 toda incursión voluntarista (o en todo caso, rozando un voluntarismo-no-voluntarista), las grietas constituyen un espacio para la nano-intervención precisamente porque la heterogeneidad estructural unida a las luchas que se libran involucrando en algunas ocasiones no solo capitales económicos y simbólicos, sino la puesta en riesgo de la propia vida, desplazan pretensión totalizadora. De este modo, todo intento de legislación $\left(\right.$ moral $^{15}$ ) respecto de las prácticas de enseñanza, investigación, publicación y organización profesional de las letras se desmorona porque lo que hay es tensión y conflicto. Lo que hay es un campo estructuralmente atravesado por los vaivenes en las políticas públicas, los desfinanciamientos estatales y la precariedad a la que los agentes oponen prácticas activistas de resistencia (tanto en formaciones como en instituciones) y de desarrollo profesional autofinanciado, a expensas, en buena parte de los casos, de su salario. ¿Cómo atreverse a osar una moral ante tal estado de las cosas?

\footnotetext{
${ }^{13}$ Uso este término con el sentido que le da Eduardo Rinesi (2003) en su teoría política, formulada entre Derrida y Shakespeare. Rinesi funda la actividad política en la explotación del "espacio de tensión que se abre entre las grietas de cualquier orden, precisamente, porque ningún orden agota en sí mismo todos sus sentidos ni satisface las expectativas que los distintos actores tienen sobre él" (p. 23). Este concepto de "política" atraviesa mis conjeturas respecto de las prácticas relativamente autónomas desarrolladas por los agentes en Argentina en el largo período recortado para esta investigación. Se trata de acciones de orden "nano", es decir, de pequeña escala, en ocasiones poco visibles, invisibilizadas o alojadas en una trama subterránea. Todas mis investigaciones pretenden, en definitiva, contribuir a exhumarlas (cf. Gerbaudo, 2016).

${ }^{14}$ Jacques Derrida (1996) emplea asiduamente esta expresión en sus escritos para dar cuenta tanto del cruce de un límite como del imperativo de no dejarse detener por una frontera cuando algo lo justifique. Se trata de un doble movimiento que no pretende borrar o anular la demarcación sino más bien interrogarla.

${ }^{15}$ Derrida (1989) diferencia la "moral", asociada a la "buena conciencia", de la "ética", asociada a la "responsabilidad": para Derrida hay "responsabilidad" cuando hay "experiencia”, cuando se está ante una "aporía”, cuando se debe afrontar el riesgo de tomar una "decisión”. En esa línea se pregunta: “es posible una experiencia que no sea experiencia de la aporía?” (1996, p. 34). Para Derrida la moral se mueve en un plano de acción regulado por lo "conforme al deber" y a lo actuado por sujeción a la "ley" regulada por el "derecho", mientras que la ética supone afrontar una "decisión" que no se limita a "poner en marcha un saber determinable o determinante" o "la consecuencia de algún orden preestablecido" (1996, p. 37). Esta ética supone actuar desde la lógica de la différance (Derrida 1967, 1972), transida por la indecidibilidad. Mientras la "moral" supone "la buena conciencia como mueca de una vulgaridad complaciente", "la forma segura de la conciencia de sí" (1996, p. 40), la "ética" ligada a la "decisión responsable" supone exponerse al "compromiso", al "riesgo absoluto" (p. 40): “Tal vez se podría sacar la conclusión de que la esencia de la decisión, aquello que la convertiría en el objeto de un saber temático o de un discurso teórico, debe permanecer indecidible: para que haya, si es que la hay, decisión" (p. 96).
} 


\section{REFERENCIAS}

Avaro, N. (2015). Pasos de un peregrino. Biografía intelectual de Adolfo Prieto. Conocimiento de la Argentina: estudios literarios reunidos (pp. 7-108). Rosario: $\mathrm{e}(\mathrm{m}) \mathrm{r}$.

Beigel, F. (2016). Científicos periféricos, entre Ariel y Calibán. Saberes institucionales y circuitos de consagración en Argentina. Dados, 3(60), 825-865.

Bekerman, F. (2013). Les instituts de recherche: expansion et reconfiguration dans le champ scientifique argentin pendant le dernier gouvernement militaire (1976-1983). Revue d'Anthropologie des Connaissances, 7(1), 247-270.

Bombini, G. (2004). Los arrabales de la literatura. La historia de la enseñanza literaria en la escuela secundaria argentina (1860-1960). Buenos Aires: Miño y Dávila.

Boschetti, A. (2009). La recomposition de l'espace intellectuel en Europe après 1945. En G. Sapiro (éd.). L'espace intellectuel en Europe. De la formation des États-nations à la mondialisation (XIX ${ }^{e}-X X I^{e}$ siècle) (pp. 147-182). Paris: La Découverte.

Bourdieu, P. (1992). Les règles de lart : genèse et structure du champ littéraire. Paris: Du Seuil.

Bourdieu, P. (1997). Les usages sociaux de la science. Pour une sociologie clinique du champ scientifique. Paris: INRA.

Bourdieu, P. (1999). Une révolution conservatrice dans lédition. Actes de la Recherche en Sciences Sociales, 126-127, 3-18.

Bourdieu, P. (2001a) Science de la science et reflexivité. Cours du Collège de France 2000-2001. Paris: Raisons d'agir.

Bourdieu, P. (2001b). Entretien: Sur l'esprit de la recherche. En Y. Delsaut et M. Rivière, (éds.). Bibliographie des travaux de Pierre Bourdieu suivi d'un entretien entre Pierre Bourdieu et Yvette Delsaut (pp. 177-239). Paris: Les Temps des Cerises.

Bourdieu, P. (2002). Les conditions sociales de la circulation internationale des idées. Actes de la Recherche en Sciences Sociales, 145, 3-8.

Bourdieu, P. (2012). Sur l'Ėtat. Cours au Collège de France (1989-1992). Paris: Raisons d'agir/Seuil.

Bourdieu, P. (2013). Manet. Une révolution symbolique. Cours au Collège de France (1998-2000) suivis d'un manuscrit inachevé de Pierre et Marie-Claire Bourdieu. Paris: Raisons d’agir/Seuil.

Caisso, C. y Rosa, N. (1987). De la constitution clandestine d'un nouvel objet. Études françaises, 23, 249-265.

CONADEP (1984). Nunca más. Informe de la Comisión Nacional sobre la desaparición de personas. Buenos Aires: Ministerio de Cultura, Programa Libros y Casas.

CONEAU (2012). La CONEAU y el sistema universitario argentino. Memoria 1996-2011. Buenos Aires: Ministerio de Educación.

Derrida, J. (1967). De la grammatologie. Paris: Minuit. 
Derrida, J. (1972). La différance. Marges de la philosophie (pp. 1-29). Paris: Minuit.

Derrida, J. (1989). Biodegradables: Seven Diary Fragments. Critical Inquiry, 4(15), 812-873.

Derrida, J. (1995). Mal d’Archive. Une impression freudienne. Paris: Galilée.

Derrida, J. (1996). Apories. Mourir-s'attendre aux "limites de la vérité". Paris: Galilée.

Desinano, N. (1965). La novelística de Manuel Gálvez. Cuadernos del Instituto de Letras, Santa Fe: UNL.

Desinano, N. (1974). El planeamiento en el área de Lengua. Rosario: Biblioteca.

Desinano, N. (1988). El discurso periodístico. Buenos Aires: Plus Ultra.

Desinano, N. (1995). Didáctica de la lengua para primero, segundo y tercer grado. Rosario: Homo Sapiens.

Desinano, N. (1997). Didáctica de la lengua para primer ciclo EGB. Rosario: Homo Sapiens.

Foucault, M. (1971) Vigilar y castigar. Nacimiento de la prisión (traducción Aurelio Garzón del Camino). Buenos Aires: Siglo XXI.

Franco, M. (2012). Un enemigo para la nación. Orden interno, violencia y "subversión", 1973-1976. Buenos Aires: Fondo de Cultura Económica.

Funes, L. (2009). Teoría literaria: una primavera interrumpida en los años setenta. Actas de las Primeras Jornadas de Historia de la Crítica en la Argentina (pp. 79-84). Buenos Aires: Facultad de Filosofía y Letras de la UBA.

Funes, L. (2011). Medievalismo en el otoño de la Edad Teórica: consideraciones parciales sobre la operación filológica. Perspectivas actuales de la investigación literaria (pp. 45-78). Buenos Aires: Editorial de la Facultad de Filosofía y Letras de la UBA.

Gerbaudo, A. (2014). La institucionalización de las Letras en la universidad argentina (1945-2010). Notas "en borrador" a partir de un primer relevamiento. Santa Fe: UNL.

Gerbaudo, A. (2016). Políticas de la exhumación. Las clases de los críticos en la universidad argentina de la posdictadura (1984-1986). Los Polvorines, Santa Fe: UNGS/UNL.

Gerbaudo, A. (2018a). El fuego, el agua, la biodegradabilidad. Apuntes metodológicos para un archivo por-venir. En D. Vigna, y P. Arán (eds.). Archivos, artes y medios digitales: teoría y práctica (pp. 41-66). Córdoba: Universidad Nacional de Córdoba.

Gerbaudo, A. (2018b). Ante un segundo ciclo de la posdictadura. El taco en la brea, 6, 4-8.

Gerbaudo, A. y Fumis, D. (2014). Esquema básico para biografías y entrevistas semiestructuradas a los agentes del campo. En A. Gerbaudo (Dir.). La institucionalización de las Letras en la universidad argentina (1945-2010) (p. 259). Santa Fe: UNL.

Giordano, A. (2011). Vida y obra: otra vuelta al giro autobiográfico. Rosario: Beatriz Viterbo. 
Girbal, N. (2007). Après la crise terminale, le système scientifique et technologique de l'Argentine (2001-2005). L'Argentine après la débâcle. Itinéraire d'une recomposition inédite (pp. 368-382). Paris: Michel Houdiard.

Giunta, A. (2009). Poscrisis. Arte argentino después de 2001. Buenos Aires: S. XXI.

Heilbron, J., Guilhot, N, y Jeanpierre, L. (2009). Internationalisation des Sciences Sociales: les leçons d'une historie transnationale. En G. Sapiro (éd.). Lespace intellectuel en Europe. De la formation des États-nations à la mondialisation (XIX ${ }^{e}-X X I^{e}$ siècle) (pp. 319-346). Paris: La Découverte.

Heilbron, J. y Gingras, Y. (2009). L'internationalisation de la recherche en Sciences Sociales et humaines en Europe (1980-2006). En G. Sapiro (éd.). Lespace intellectuel en Europe. De la formation des États-nations à la mondialisation (XIX ${ }^{e}-X X I^{e}$ siècle) (pp. 39-390). Paris: La Découverte.

Pucciarelli, A. (2011). Los años de Menem. La construcción del orden neoliberal. Buenos Aires: S. XXI.

Rinesi, E. (2003). Politica y tragedia. Hamlet entre Hobbes y Maquiavelo. Buenos Aires: Colihue.

Sapiro, G. (1999). La Guerre des écrivains (1940-1953). Paris: Fayard.

Sapiro, G. (2012). International Cooperation in the Social Sciences and Humanities: Comparative Socio-Historical Perspectives and Future Possibilities. Paris: INTERCO-SSH. Disponible en http://interco-ssh.eu/project-description/

Sapiro, G. (2017). Introduction, Développement professionnel et évolutions du métier décrivain, Devenir écrivain-e: de la reconnaissance symbolique à la reconnaissance professionnelle. En G. Sapiro, y C. Rabot (éds.). Profession? Écrivain (pp. 7-16; 19-41; 43-76). Paris: CNRS Éditions.

Sapiro, G., Heilbron, J., Boncourt, T. y Sorá, G. (2013). Indicators of the Internationalization of an academic discipline in Social Sciences and Humanities. Paris: INTERCO SSH. Disponible en http://interco-ssh.eu/en/

Sorá, G. y Beigel, F. (2019). Arduous Institutionalization in Argentina's SSH: Expansion, Asymmetries and uncertain future. En C. Fleck (éd.). Shaping Human Science Disciplines: Institutional Developments in Europe and Beyond (pp. 327-360). London: Palgrave-MacMillian.

Williams, R. (1977). Marxism and literature. Oxford: Oxford University Press. 